\title{
Kinova Modular Robot Arms for Service Robotics Applications
}

\author{
Alexandre Campeau-Lecours, Laval University, Quebec, Canada \\ Hugo Lamontagne, Kinova, Boisbriand, Canada \\ Simon Latour, Kinova, Boisbriand, Canada \\ Philippe Fauteux, Kinova, Boisbriand, Canada \\ Véronique Maheu, Kinova, Boisbriand, Canada \\ François Boucher, Kinova, Boisbriand, Canada \\ Charles Deguire, Kinova, Boisbriand, Canada \\ Louis-Joseph Caron L’Ecuyer, Kinova, Boisbriand, Canada
}

\begin{abstract}
This article presents Kinova's modular robotic systems, including the robots JACO2 and MICO2, actuators and grippers. Kinova designs and manufactures robotics platforms and components that are simple, sexy and safe under two business units: Assistive Robotics empowers people living with disabilities to push beyond their current boundaries and limitations while Service Robotics empowers people in industry to interact with their environment more efficiently and safely. Kinova is based in Boisbriand, Québec, Canada. Its technologies are exploited in over 25 countries and are used in many applications, including as service robotics, physical assistance, medical applications, mobile manipulation, rehabilitation, teleoperation and in research in different areas such as computer vision, artificial intelligence, grasping, planning and control interfaces. The article describes Kinova's hardware platforms, their different control modes (position, velocity and torque), control features and possible control interfaces. Integration to other systems and application examples are also presented.
\end{abstract}

\section{KEYWORDS}

Assistive Robotics, Force Control, JACO, Kinova, MICO, Physical Human-Robot Interaction, Robotics, Service Robotics, Torque Control

\section{INTRODUCTION}

Robots are well implemented in industry for manufacturing and are used in a large array of applications. Their speed, precision, stamina and strength allow them to perform many tasks better and faster than humans. They also allow humans to avoid many tasks that would be too dangerous or repetitive. These industrial robots typically have a limited number of sensors and decision capabilities preventing them from safely operating near humans. However, in many applications, humans would benefit from a direct interaction with robots. For instance, we could interact with the robot by taking advantage of our own capabilities (capacity of decision) along with the robot's capabilities (strength, endurance, 
stamina) thus creating a synergy allowing to accomplish tasks that were not possible for one or the other alone. In order for this new collaboration to be possible, robots must be safe, intuitive and have a better understanding of their environment (De Santis et al, 2008; Haddadin and Croft, 2016). In order to achieve this, sensor information and advanced algorithms are very important.

This new era of human assistance is emerging in many applications. In manufacturing, robots work closely with operators in the same workspace. For example, this includes collaborative devices and new commercial robots from companies such as Kuka, Rethink Robotics, Universal Robots, $\mathrm{ABB}$, Kawada Industries and MABI.

\section{Kinova's History}

Kinova was founded in 2006 as a company for development of innovative solutions for compensating the loss of mobility of upper limbs. The inspiration came from Kinova's CEO and co-founder's uncle, who suffered from muscular dystrophy, as did two of his brothers. He was challenged by the idea of developing an arm which could be controlled by his only active finger and allow him to become more independent and able to grasp and manipulate objects in his surroundings without external assistance. Kinova then designed the robot JACO (as shown in Figure 1) to assist people with physical disabilities (Campeau-Lecours et al., 2016). From this experience, the company developed other technologies, such as the robot MICO, in the broader area of human assistance and service robotics. These robots are now sold in more than 25 countries.

The latest versions, $\mathrm{JACO}^{2}$ and $\mathrm{MICO}^{2}$ are 6 degrees of freedom (DOF) lightweight robots that are used in many applications. Kinova's actuators are modular and can also be used to design robots with different configurations. Each actuator is equipped with many sensors such as position, torque, accelerometers and temperature and can perform multiple rotations. The robots can be controller in position, velocity and torque. The robots also use an embedded controller located in the robot base enabling a compact design and easy integrations. The company operates in two main markets, namely Service Robotics and Assistive Robotics.

\section{Service Robotics}

Service robotics (see Figure 2) aims to assist human beings to accomplish different tasks. The International Federation of Robotics (IFR) has proposed a tentative definition, "A service robot is

Figure 1. JACO mounted on a powered wheelchair. Figure courtesy of Kinova.

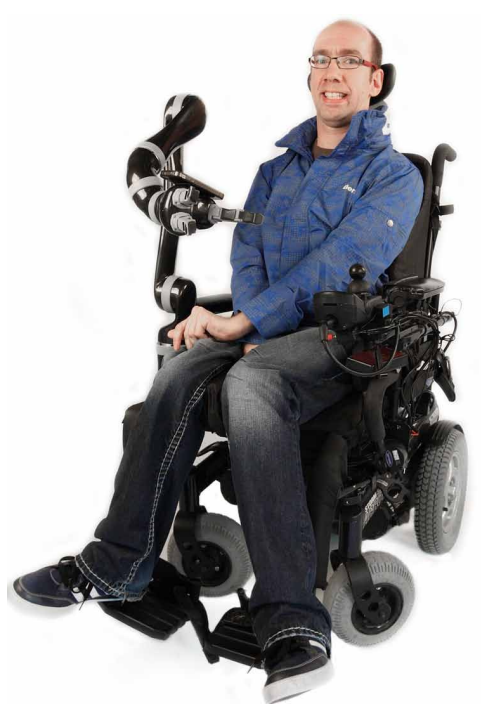


Figure 2. Two JACO in a torso configuration. Husky Manipulator from Clearpath Robotics with JACO integration. Figure courtesy of Clearpath Robotics.

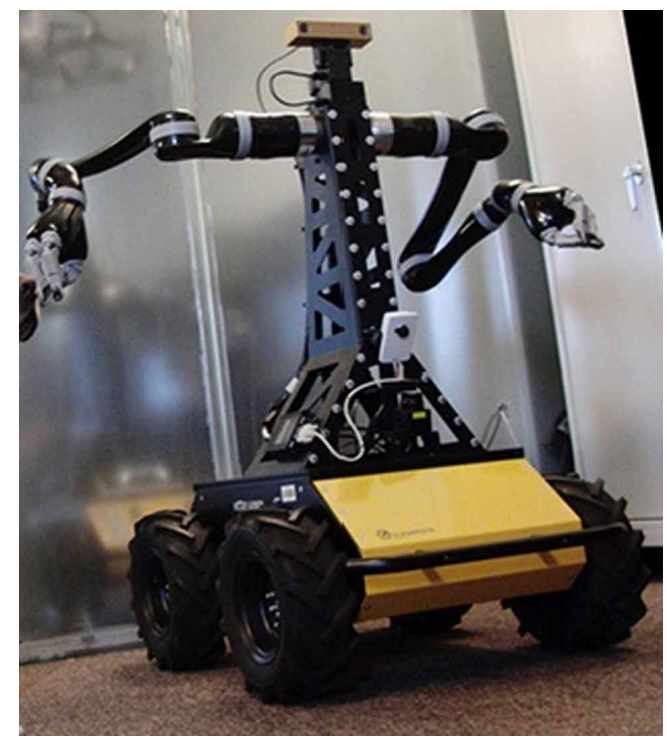

a robot which operates semi- or fully autonomously to perform services useful to the well-being of humans and equipment, excluding manufacturing operations." In these applications, safety, design and environment awareness are a prime concern and $\mathrm{JACO}^{2}$ and $\mathrm{MICO}^{2}$ were designed accordingly.

\section{Assistive Robotics}

Robots for assisting in manipulation become a powerful solution for enhancement of autonomy for people living with severe upper-limb motor impairments. Such robots may assist people in various tasks such as eating, drinking, moving various objects, turning book pages, opening and closing doors, turning on and off electrical and electronic systems. It also serves to move the user's hand or feet, to cross legs or to stretch and is even used as a rehabilitation device in clinics. Such assistive devices must be lightweight, multifunctional, intuitive and easy to integrate. $\mathrm{JACO}^{2}$ and $\mathrm{MICO}^{2}$ empower people living with disabilities to push beyond their current boundaries and limitations (CampeauLecours et al., 2016).

\section{Paper Structure}

The paper is structured as follows. Kinova's hardware, including robots $\mathrm{JACO}^{2}$ and $\mathrm{MICO}^{2}$, actuators and grippers are presented along with their specifications and features. The different control interfaces to interact with Kinova's products are then detailed. Finally, the control modes, including position, velocity and torque control, are presented. An emphasis is put on the torque controller algorithms and features. Finally, integration of Kinova's technology with other products are presented along with application examples.

\section{KINOVA'S HARDWARE SPECIFICATIONS}

\section{$\mathrm{JACO}^{2}$ and $\mathrm{MICO}^{2}$ Robots}

Kinova's actuators are modular and can thus be used to build different robot configurations. However, classic configurations are offered with a fully integrated controller, including Cartesian and angular 
control (position, velocity and force/torque), intelligent singularity and joint limitation avoidance system, trajectories, gravity estimation and force control schemes. The classic configurations include $\mathrm{JACO}^{2}$ and $\mathrm{MICO}^{2} 6$ DOF versions (shown in Figure 3) and $\mathrm{JACO}^{2}$ and $\mathrm{MICO}^{2} 4$ DOF versions (shown in Figure 4). 7 DOF versions are also planned to be released. Table 1 shows specifications of these robots. $\mathrm{MICO}^{2}$ uses actuators K-75 for the first three joints while $\mathrm{JACO}^{2}$ uses K-75 actuators for joint 1 and 3 and a K-75+ actuator for joint 2. Both robots use K-58 actuators for the wrist. Kinova's actuators and their specifications are presented in section 2.3.

A distinct feature of Kinova's robots is that the controller is integrated in the robot's base thus leading to a very compact design. Because there is no large and cumbersome controller, the robot can easily be installed anywhere and can easily be used in mobile applications. The robot can be powered by a standard power outlet or through a battery. The actuators can perform multiple rotations which is very useful in many applications. $\mathrm{JACO}^{2}$ and $\mathrm{MICO}^{2} 6 \mathrm{DOF}$ versions feature a special wrist

Figure 3. $\mathrm{MICO}^{2}$ and $\mathrm{JACO}^{2}$ 6DOF versions side by side. Figure courtesy of Kinova.

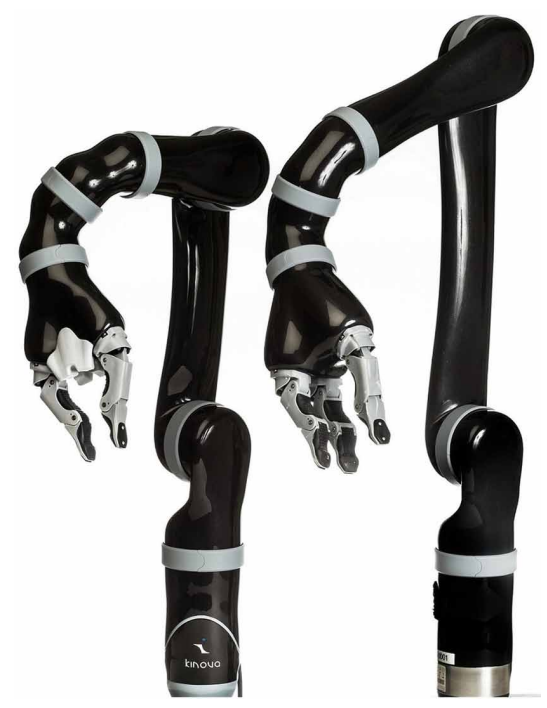

Figure 4. $\mathrm{JACO}^{2}$ and $\mathrm{MICO}^{2} 4 \mathrm{DOF}$ versions side by side. Figure courtesy of Kinova.

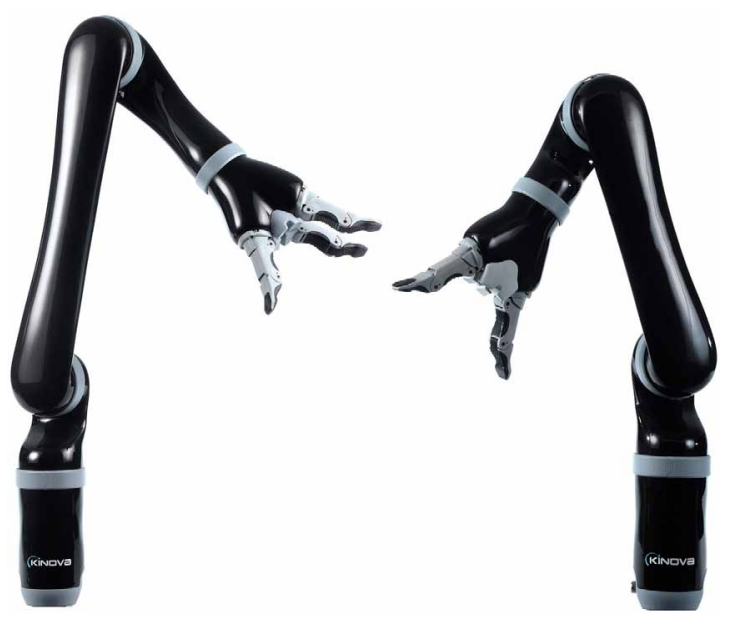


Table 1. $\mathrm{JACO}^{2}$ and $\mathrm{MICO}^{2}$ specifications. The values are for robots with no gripper.

\begin{tabular}{|c|c|c|c|c|c|}
\hline & & \multirow{2}{*}{$\begin{array}{l}\mathrm{JACO}^{2} \\
6 \mathrm{DOF}\end{array}$} & \multirow{2}{*}{$\begin{array}{l}\mathrm{MICO}^{2} \\
6 \mathrm{DOF}\end{array}$} & \multirow{2}{*}{$\begin{array}{l}\mathrm{JACO}^{2} \\
4 \mathrm{DOF}\end{array}$} & \multirow{2}{*}{$\begin{array}{l}\mathrm{MICO}^{2} \\
4 \mathrm{DOF}\end{array}$} \\
\hline & Unit & & & & \\
\hline Maximum Reach & $\mathbf{c m}$ & 90 & 70 & 75 & 55 \\
\hline \multicolumn{6}{|l|}{ Payload: } \\
\hline Mid-range continuous & $\mathrm{kg}$ & 2.6 & 2.1 & 4.4 & 5.2 \\
\hline Full-reach peak/temporary & $\mathrm{kg}$ & 2.2 & 1.5 & 3.5 & 2.7 \\
\hline Links material & & Carbon fiber & Reinf. plastic & Carbon fiber & Reinf. plastic \\
\hline Total weight & $\mathrm{kg}$ & 4.4 & 4.6 & 3.6 & 3.8 \\
\hline Maximum linear speed & $\mathrm{cm} / \mathrm{s}$ & \multicolumn{4}{|l|}{20} \\
\hline Power supply voltage & VDC & \multicolumn{4}{|l|}{18 to 29} \\
\hline Average power & $\mathrm{W}$ & \multicolumn{4}{|l|}{25} \\
\hline Standby power & $\mathrm{W}$ & \multicolumn{4}{|l|}{5} \\
\hline Peak Power & $\mathrm{W}$ & \multicolumn{4}{|l|}{100} \\
\hline \multicolumn{6}{|l|}{ Control frequency: } \\
\hline High level API & $\mathrm{Hz}$ & \multicolumn{4}{|l|}{100} \\
\hline Low level API & $\mathrm{Hz}$ & \multicolumn{4}{|l|}{500} \\
\hline
\end{tabular}

configuration shown in Figure 3. This wrist is designed for safety to physically prevent any zones where a user's limb could be pinched or squeezed.

$\mathrm{JACO}^{2}$ and $\mathrm{MICO}^{2}$ can be built with different options (for instance a 3-fingers or 2-fingers gripper) and controlled with different interfaces as described in the next sections. Custom versions of both devices can also be designed.

\section{Sensors}

Kinova's robots are equipped with a variety of sensors including:

Sensors in the base of the arm:

$$
\begin{array}{ll}
\text { - Supply voltage } \\
\circ \quad \text { Temperature } \\
\circ \quad \text { Accelerometer XYZ }
\end{array}
$$

Sensors in each actuator:

$$
\begin{array}{ll}
\circ & \text { Optical position encoder } \\
\circ & \text { Absolute position } \\
\circ & \text { Torque } \\
\circ & \text { Current } \\
\circ & \text { Temperature } \\
\circ & \text { Accelerometer XYZ }
\end{array}
$$

Sensors in each fingers:
- Current
- Temperature
- Optical position encoder 


\section{Actuators}

Kinova's actuators mechanics and electronics were specifically designed for $\mathrm{JACO}^{2}$ and $\mathrm{MICO}^{2}$ requirements. They use DC brushless motors with Harmonic Drive technology and are equipped with a set of sensors such as encoders, torque sensor, current sensor, temperature sensor and accelerometers. They can be controlled in position or torque and can perform multiple rotations thanks to an integrated slip ring technology. The actuators are independent, interoperable and can be used to build custom robots or systems (see Figure 5). Actuators specifications are presented in Table 2.

The gripper design is very important both in service and assistive robotics since it must be able to pick objects of daily living, both varying in shape and size. Additionally, other parameters must be considered, such as the size, the gripping force, the durability, the gripping speed and the achievable grasps. Underactuated grippers (Birglen et al., 2008), with one motor per finger, where thus designed to simplify the controller, to reduce costs and to automatically adapt to the shape of different objects. Kinova's grippers include a 2-finger (KG-2) and a 3-fingers (KG-3) hand as shown in Figure 6. The specifications are shown in Table 3. $\mathrm{JACO}^{2}$ and $\mathrm{MICO}^{2}$ can be configured with the KG-2 or KG-3 gripper, or even with no gripper at all (if for instance the client does not need a gripper or if he wants to install its own end-effector). To this end, four wires are available

Figure 5. Kinova's actuators: K-75+, K-75 and K-58. Figure courtesy of Kinova.
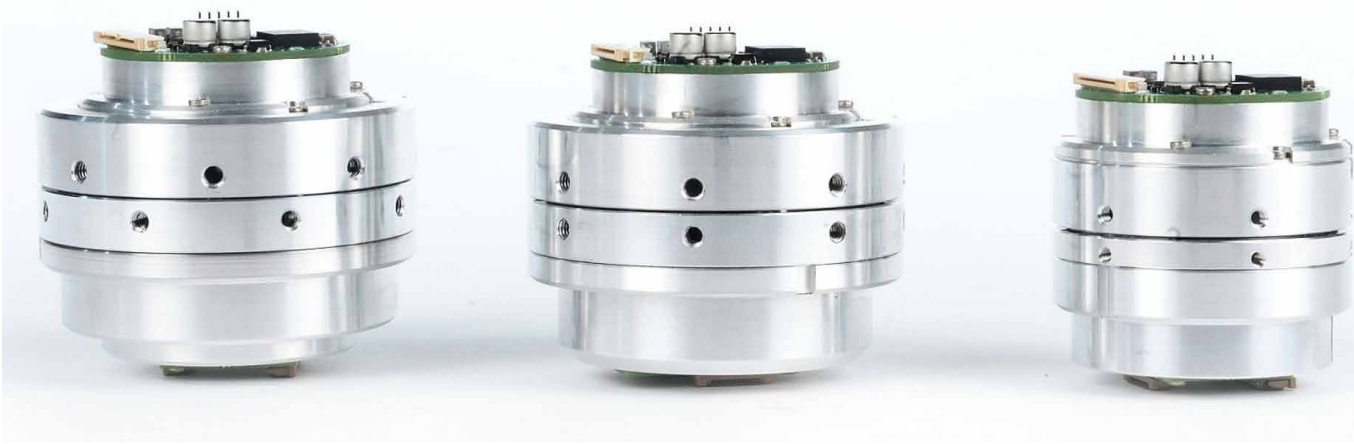

Table 2. Kinova's actuators specifications

\begin{tabular}{|c|c|c|c|c|}
\hline & Unit & K-75 + & K-75 & K-58 \\
\hline Nominal torque & $\mathrm{Nm}$ & 12.0 & 9.2 & 3.6 \\
\hline Peak Torque & $\mathrm{Nm}$ & 30.5 & 18.0 & 6.8 \\
\hline No load speed & $\mathrm{rpm}$ & 12.2 & 9.8 & 20.3 \\
\hline Nominal speed & $\mathrm{rpm}$ & 9.4 & 7.0 & 15.0 \\
\hline Weight & $\mathrm{g}$ & 570 & 587 & 357 \\
\hline Reduction ratio & & 136 & 160 & 110 \\
\hline Angular Range (software limited) & turns & \multicolumn{3}{|l|}{ \pm 27.7} \\
\hline Communication protocol & & \multicolumn{3}{|l|}{ RS485 } \\
\hline \multicolumn{5}{|l|}{ Control frequency: } \\
\hline High level API & $\mathrm{Hz}$ & \multicolumn{3}{|l|}{100} \\
\hline Low level API & $\mathrm{Hz}$ & \multicolumn{3}{|l|}{500} \\
\hline
\end{tabular}


Figure 6. Kinova's grippers: KG-2 and KG-3. Figure courtesy of Kinova.
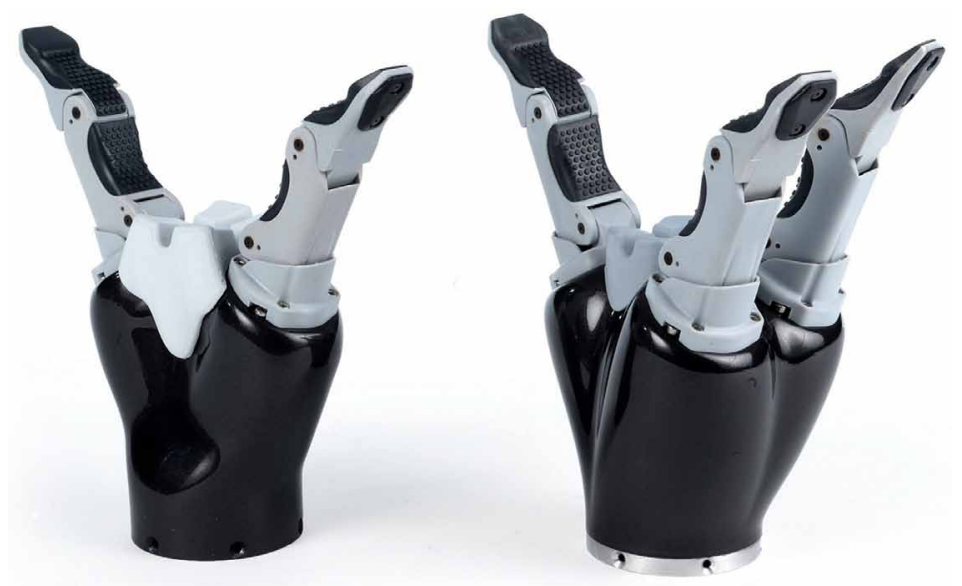

Table 3. Kinova's gripper specifications

\begin{tabular}{|l|l|l|l|}
\hline & \multicolumn{1}{|c|}{ Unit } & \multicolumn{1}{c|}{ KG-2 } & \multicolumn{1}{c|}{ KG-3 } \\
\hline Number of fingers & & 2 & 3 \\
\hline Weight & $\mathrm{g}$ & 556 & 727 \\
\hline Opening (fingertip) & $\mathrm{mm}$ & 175 & 175 \\
\hline Grip force & $\mathrm{N}$ & 25 & 40 \\
\hline
\end{tabular}

at the motor output: $24 \mathrm{~V}$ and Ground for power and two wires that can be used for different communication protocols (allowing to control the end-effector through the arm without having to install external wires). Kinova's grippers can also be installed on other robots or systems (as shown on a Baxter robot in Figure 7).

\section{Custom Robot}

While it is possible to configure $\mathrm{JACO}^{2}$ and $\mathrm{MICO}^{2}$ with different options, it is also possible to use actuators and grippers separately to build custom robot configurations or systems. Figure 8 shows an example of a planar RRRR robot with a KG-2 gripper.

\section{Extensions}

Extensions to install additional hardware on the robot are also available. For example, a ring can be installed on the actuators, at the elbow or at the wrist, in order to install cameras (as shown in Figure 9).

\section{INTERFACE}

In order to meet different needs, different interfaces can be used to control the robots such as a Software Development Kit (SDK) (including a high-level API, a low-level API and a Development center), a ROS package and a Joystick. The different interface options are shown in Figure 10. 
Figure 7. Kinova's gripper KG-3 installed on a Baxter robot. Figure courtesy of Rethink Robotics.

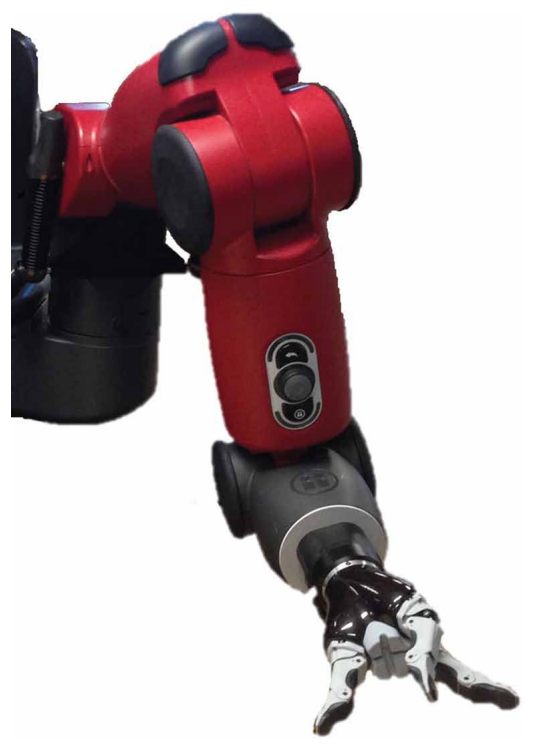

Figure 8. Custom planar RRRR robot. Figure courtesy of Kinova.

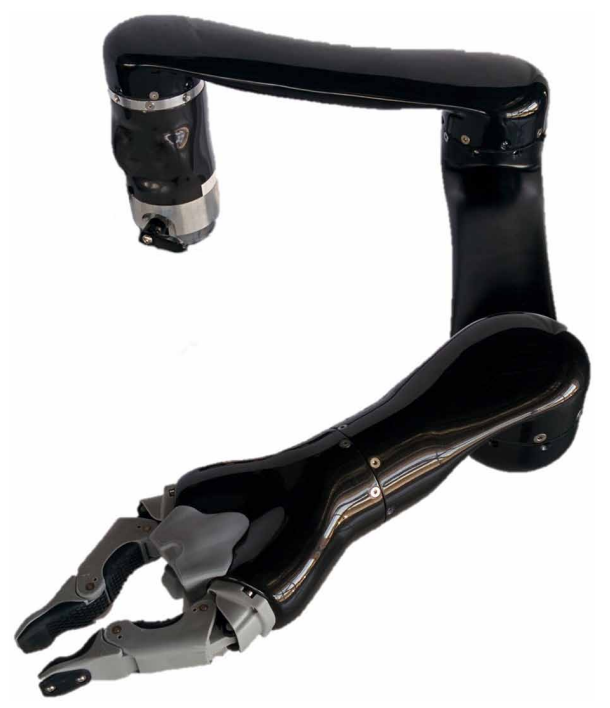

\section{Kinova SDK - Development Center}

The Development center is a complete set of interface, documentation, examples and software tools that help developers configure and interact with any Kinova product. It is available under Windows and Ubuntu. The main page is shown in Figure 11.

\section{Kinova SDK - High-Level API}

The high-level API allows to integrate the robot with the developer's system and to configure the robot's parameters. For instance, it allows getting information from the robot (torque, current, 
Figure 9. A Microsoft Kinect attached to JACO2 wrist. Figure courtesy of University of Colorado Boulder, Correll Robotics Lab.

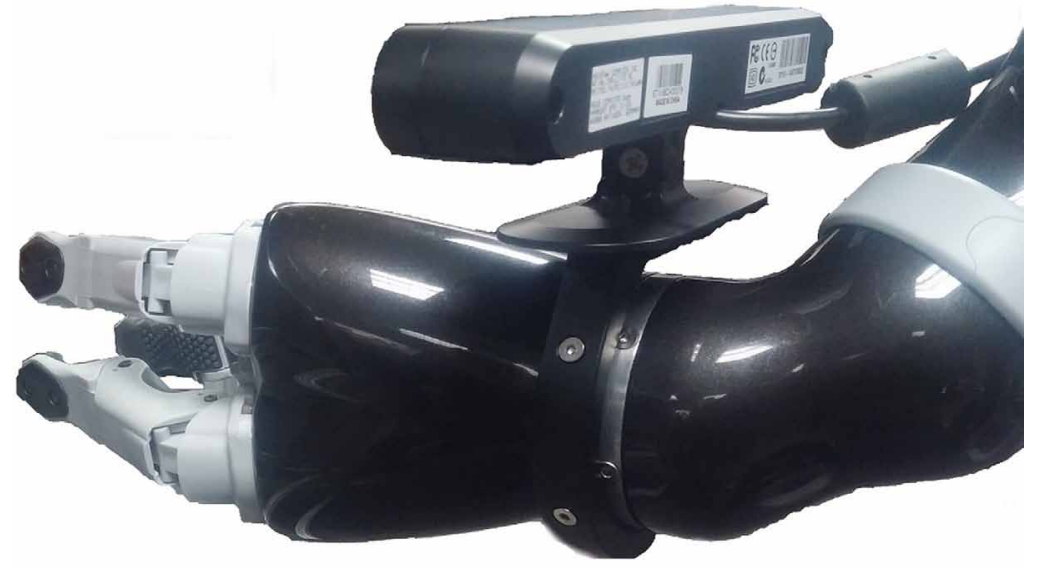

Figure 10. Possible control interfaces. Figure courtesy of Kinova.

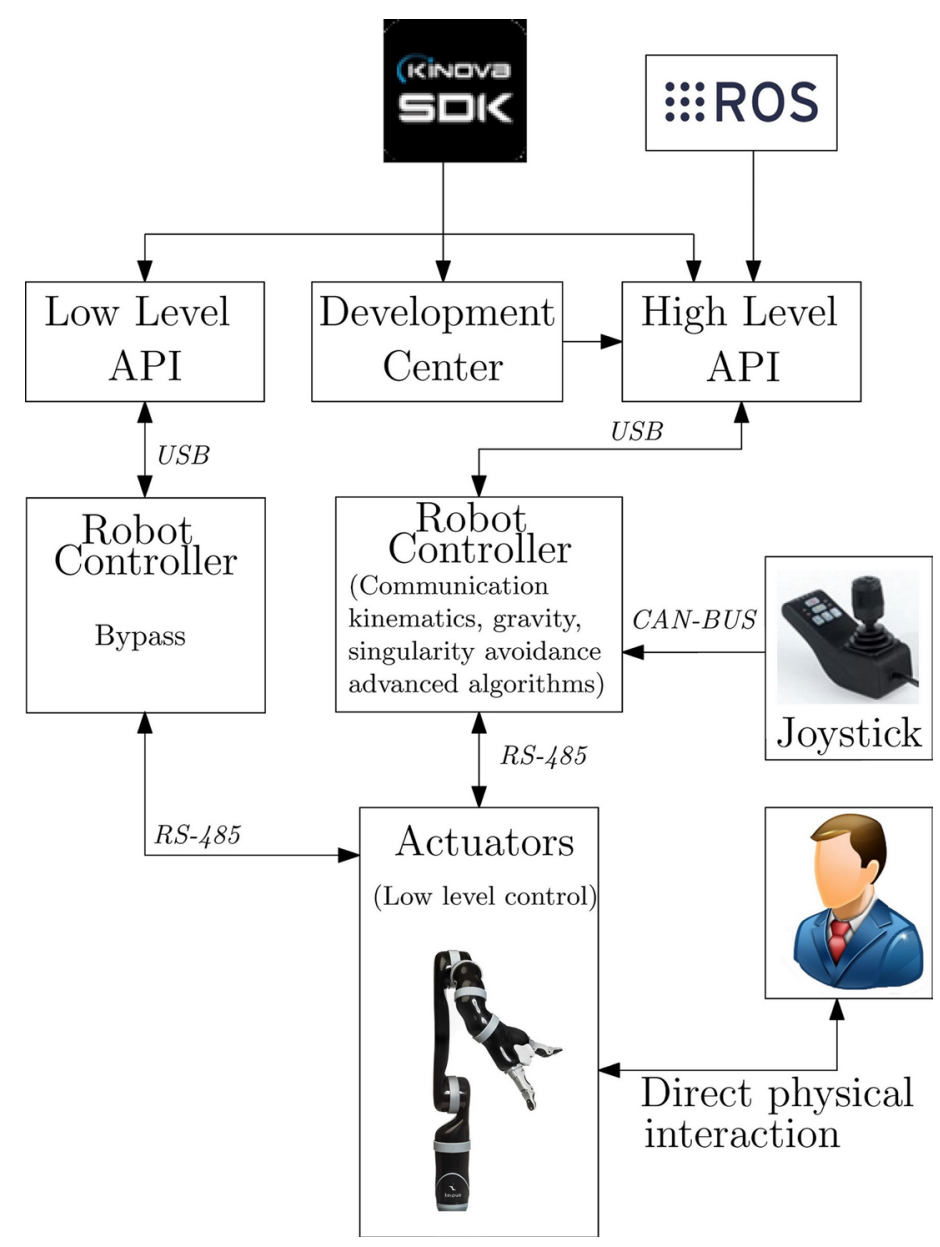


Figure 11. Development center main page. Figure courtesy of Kinova.

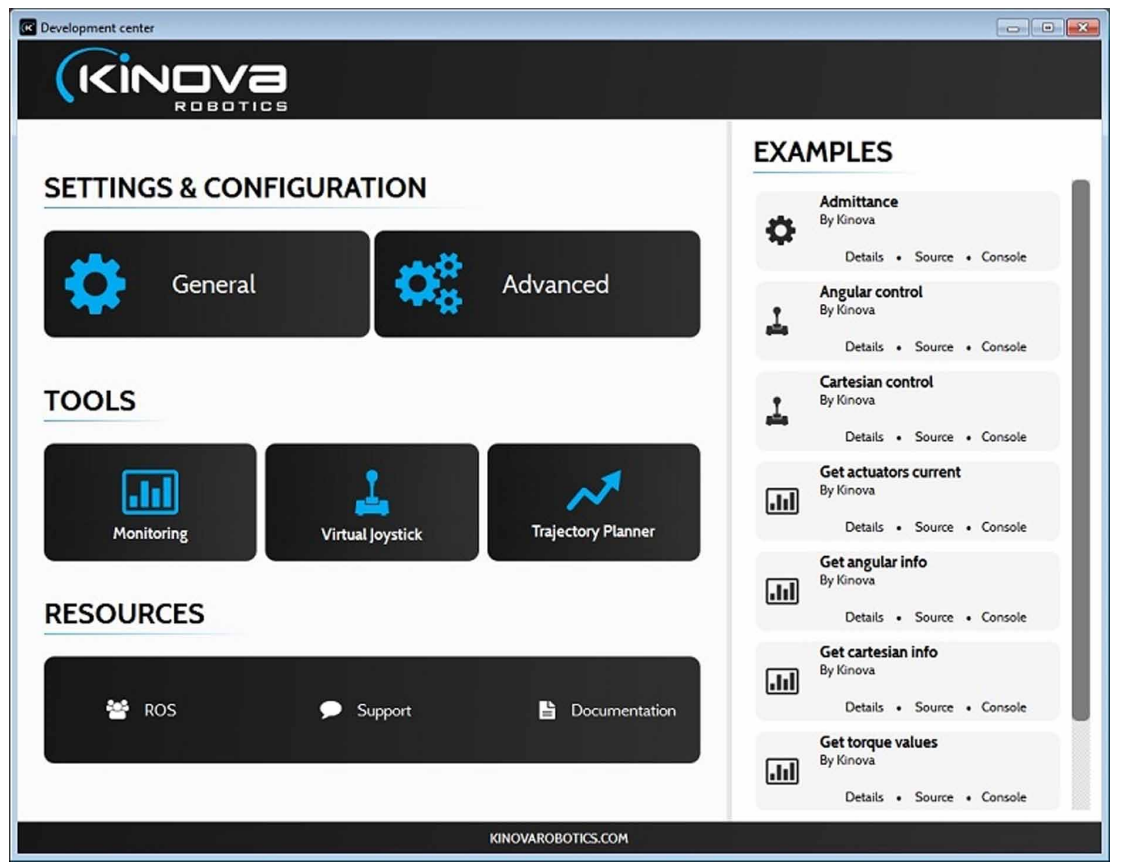

position, etc.) and sending commands (position, velocity or torque) both in angular and Cartesian mode. The high-level API uses Kinova's main controller and developers can then take advantage of advanced algorithms that are already included in the Kinova controller library such as Cartesian control, intelligent singularity and joint limitation avoidance system, trajectories, gravity estimation and force control schemes. The robot controller operates at $100 \mathrm{~Hz}$. The high-level API can be used in $\mathrm{C}++$ applications, both under Windows and Ubuntu.

\section{Kinova SDK - Low-Level API}

Although the high-level API allows controlling the robot with the Kinova high-level controller library, the low-level API may be more suitable for some developers. This low-level API allows to communicate directly with the actuators, allowing more flexibility and a higher refresh rate of $500 \mathrm{~Hz}$.

\section{R.O.S.}

The Robot Operating System (ROS) is an open source set of software libraries and tools that help building robot applications. It includes, algorithms, development tools and allows standardizing the development and the integration of robot applications.

\section{Joystick}

Kinova's joystick enables the user to quickly operate the robot. It has a three degrees of freedom stick and 7 buttons. The basic mapping allows performing translations, rotations and moving the fingers.

\section{Control}

$\mathrm{JACO}^{2}$ and $\mathrm{MICO}^{2}$ have two main control modes, as shown in Figure 12: trajectory control (including position, velocity and admittance force control) and torque control (including angular torque control and Cartesian force/torque control). 


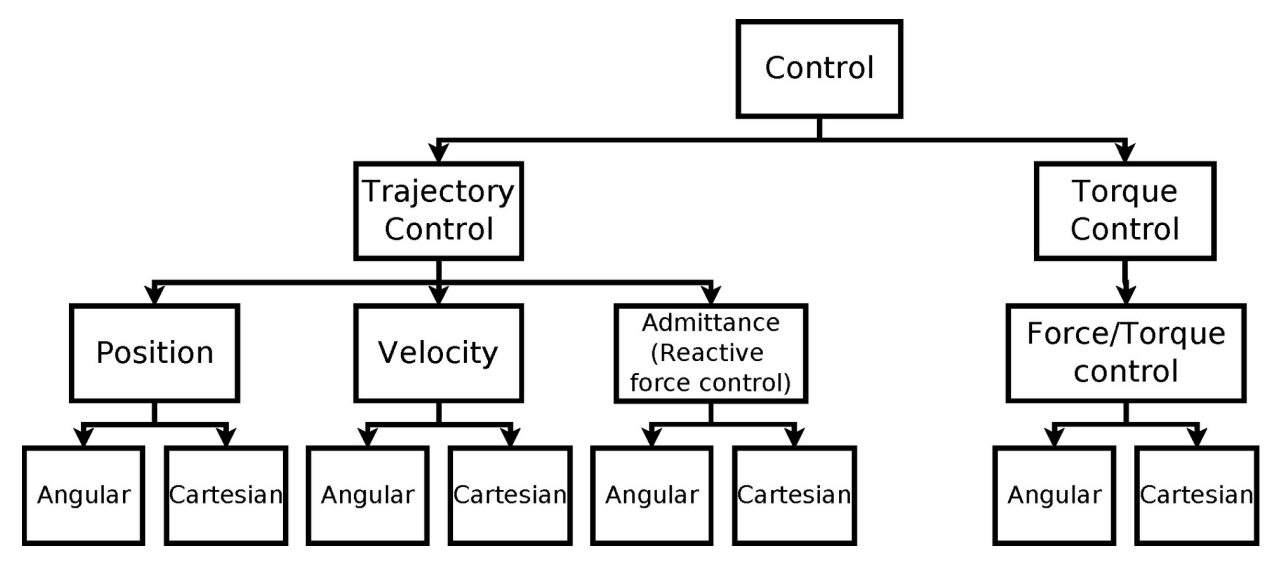

\section{Trajectory Control}

\section{Position}

The user can send a simple position to reach or define multi-point trajectories. This can be done in angular mode (where the position of each joint must be defined) or in Cartesian mode (where the desired translation (XYZ) and the desired orientation (Euler $\Theta X Y Z$ must be defined). The user interface uses Euler angles while the internal computations are done with Quaternions.

\section{Velocity}

The user can also send velocity commands both in angular or Cartesian mode. As a safety feature, in case of communication problems, the robot will stop if it has not received a command for $10 \mathrm{~ms}$. The user must specify the translation velocity (XYZ) and the orientation velocity (instantaneous velocity around the end effector frame).

\section{Intelligent Singularity and Joint Limitation Avoidance System}

Singularities and joint limitations are present in every robot and may prevent some motions. In an industrial application, the trajectories would be planned to avoid these zones or the robot would otherwise stop when reaching those limits. However, in service robotics and in human-robot interaction applications, trajectories are defined in real time by the user. Singularities and joint limitations are thus frequently reached and it would be very difficult to control the robot if it stopped each time it encountered a limitation.

A singularity and joint limit sliding algorithm was thus developed. Instead of stopping on the limitation, the robot will instead slide on it. For example, in a given configuration, a desired downward motion could require that an actuator reaches its position limit. Instead of stopping the motion, the algorithm will allow a very small forward motion, to allow the actuator to move away from the limitation, in order to let the robot continue its downward motion. The robot would not perform a perfect vertical motion but the algorithm makes the robot very intuitive to use with smooth and continuous motion.

\section{Admittance Force Control}

Impedance and admittance controllers are two types of force control algorithms. Impedance controllers accept a displacement as input (which is measured) and react with a force. Conversely, admittance controllers accept a force as input (which is measured) and react with a displacement. The mapping 
between the force and the displacement is normally a mass-damper-spring model. For a detailed description of admittance control, see (Lecours et al., 2012; Lecours and Gosselin, 2013).

The algorithm implemented in $\mathrm{JACO}^{2}$ and $\mathrm{MICO}^{2}$ uses the torque sensors available in every actuator and the robot can then react to forces applied on any of its links (and not only at the endeffector). Typical applications include manual trajectory teaching and safety features (to limit the force that the robot can apply or to be able to manually stop a motion). While admittance control can be used to react to a force input, it cannot be used to exert a prescribed force on the environment.

\section{Torque Control}

While position control is widely used in robotics, it is not suitable for many applications (Villani and De Schutter, 2008). Indeed, many tasks require the robot to apply a prescribed force on the environment or to handle physical contacts between the robot and the environment. Additionally, force control schemes become mandatory to achieve robust and versatile behavior as well as safe and dependable operation in the presence of humans (Bicchi et al., 2008).

Angular torque commands and Cartesian force commands can be sent to the robot/actuators through the high-level API and the low-level API. By using the high-level API, the user takes advantage of advanced algorithms implemented in the robot controller (described in the next sections) such as gravity estimation, vibration controller, Cartesian force control and multi-actuator mode switch management. The general torque control scheme is shown in Figure 13.

\section{Force/Torque Control}

Torque commands can be sent to each individual actuator. Cartesian forces commands (linear XYZ) and torque commands (rotation XYZ) can also be sent to the robot. As a safety feature, the robot will stop if it has not received a command for a given amount of time.

\section{Figure 13. Torque control architecture. Figure courtesy of Kinova.}

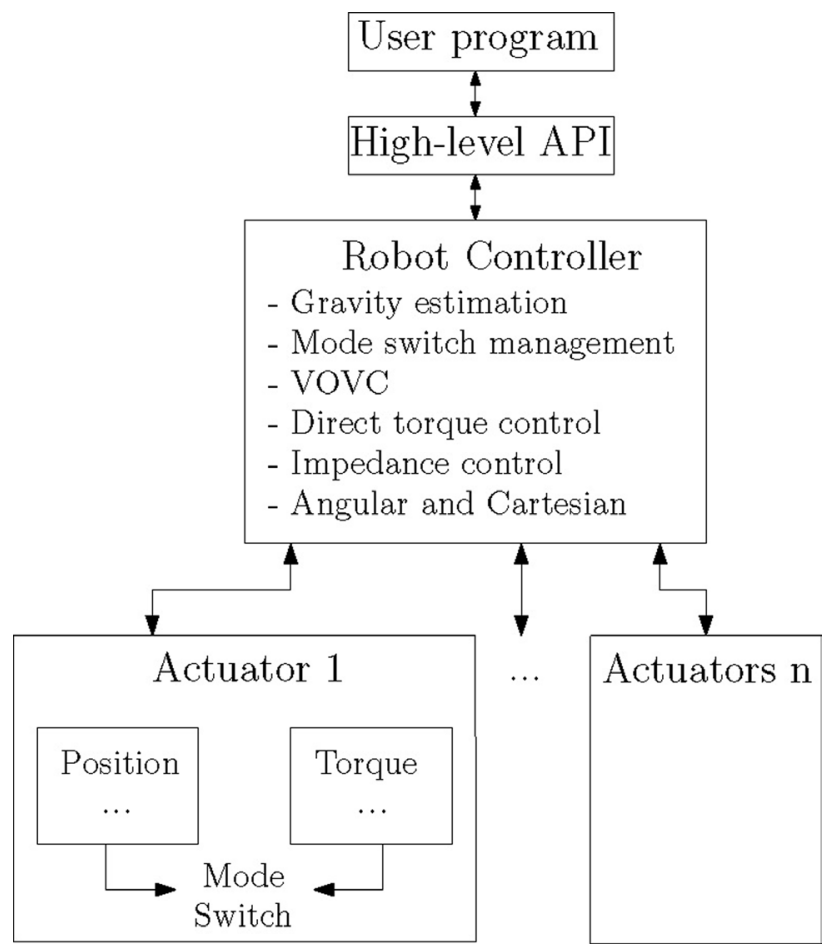


By default, when operating in torque control mode, gravity and friction are compensated for. The robot is thus in a floating mode where it maintains its position and will move freely if a force is applied on any of its link.

\section{Mode Switching Management}

From position to torque: In order to successfully switch from position to torque mode, all actuators torque command must be within a given range (customizable) from the measured torque value. This is a safety feature to ensure that the robot will not move abruptly after a mode switch.

From torque to position: When switching from torque to position mode, the actuators desired position is set to the measured position to obtain a smooth transition. As a safety feature, if a fault is observed while in torque control mode, the actuators will automatically switch to position mode to stop the robot (thus acting as a brake).

\section{Safety Features}

Additional safety features are implemented in the torque control mode:

- Maximum velocity management: When researching, developing or implementing robotic algorithms, errors are relatively easy to make. To this end, a velocity safety feature was introduced: if the velocity of an actuator exceeds a prescribed threshold, all actuators will switch to position control and will thus stop. A safety factor, (varying between 0 and 1 ) is used to define the velocity threshold. A safety factor of 0.3 means that the robot will stop if an actuator velocity exceeds $30 \%$ of its maximum velocity while a safety factor of 1 means that the feature is deactivated. A low value is recommended when developing the program and a higher value can be set when the program is developed and tested;

- Maximum position management: Each actuator has a minimum and maximum position that they can reach. For instance, $\mathrm{JACO}^{2}$ and $\mathrm{MICO}^{2}$ actuators 2 and 3 position must be limited to avoid collisions with the robot links. To this end, a virtual wall (described next) will repulse the actuators from the limitation. However, if an actuator still reaches its limitation, all actuators will switch back to position control mode and the robot will stop;

- Vibration observer / controller (VOVC): It is well known that several conditions can induce vibrations or oscillations when using force control schemes (time delay, interaction stiffness, friction, etc.) (Hannaford and Ryu, 2008) and a vibration observer/ controller was thus implemented. The main objective is to adjust the controller to reduce or eliminate the high-frequency/amplitude oscillations. The algorithm first observes the velocity to detect all the signal local maxima and minima. Based on the difference of time and amplitude between these extrema, a vibration index is obtained. From this vibration index, the controller then reduces the control gains or adds damping to reduce the vibration level. The vibration controller is only active when the vibration index is over a given threshold and it does not influence the controller under normal operating conditions. The algorithm is robust to signal noise and requires only low computational costs. The VOVC sensitivity to vibrations can be changed through the API and can also be turned off;

- Robot protection zone: While limiting actuators maximum position prevents some collisions, it does not prevent them all. Indeed, some combination of actuator positions can lead to collisions with the robot links. Because such collisions may occur rapidly when developing torque control algorithms, a basic protection zone was implemented. Near a collision point, damping is added to the actuators to slow down the motion and if the distance to the collision point continues to decrease, the robot will stop by switching back to position mode. 


\section{Gravity Management}

Gravity management is very important for the force control schemes and different options were thus implemented:

- Gravity vector: The gravity vector, [gx, gy, gz] is used to indicate the orientation of the robot's base according to the gravity direction. For instance, if the robot is standing, as in Figure 3, the gravity vector would be $[0,0,-9.81]$. If a user does not want the provided gravity compensation (for instance to compute its own compensation), the values can all be set to zero;

- Gravity input parameters mode: Different gravity estimation modes are available. The first mode consists of giving all the links parameters (mass and centre of mass in XYZ). The second mode consists of giving the parameters obtained from a linearization of the gravity model. The latter are obtained through a least square identification of the parameters which lead to a better estimation. In order to obtain the specific parameters for each robot, an identification sequence can be launched through the high-level API (the robot will perform a trajectory and the algorithm will find the optimal parameters);

- Payload: The payload mass and centre of mass must be accounted for by the gravity model. To that end, the user can specify the payload parameters through the API. An automatic identification sequence is also planned to be implemented.

\section{Low Level Actuator Control}

The actuators microcontroller manages the low-level position and torque control. The general torque control scheme is shown in Figure 14. The inputs to the actuator are the desired torque $\left(\tau_{\mathrm{d}}\right)$, the vibration controller output (VOVC) and configurations parameters (such as controller gains, safety factor, filter values, etc.).

The first level consists of a controller with feedforward which computes a current to send to the motors. Because the motors are controlled with a voltage PWM, this current is converted into a desired voltage. Some features are described below:

- Error observer: As explained above, if the velocity exceeds a given threshold or if the position exceeds a given limit, the actuator will switch to position control. Additionally, if no command is sent to the actuator for a given amount of time, the actuator will also stop by switching to position mode;

- Joint damping: Damping can be added to the actuators. This feature is useful in different applications, for instance to increase the stability margin or to reduce vibrations;

- Virtual wall: Some actuators maximum position must be limited to prevent collisions with the robot links. It would be very annoying if the robot stopped every time it reached a limit. To this end, a virtual spring-damper wall was implemented on each joint limitation. When reaching a

Figure 14. Low level torque control. Figure courtesy of Kinova.

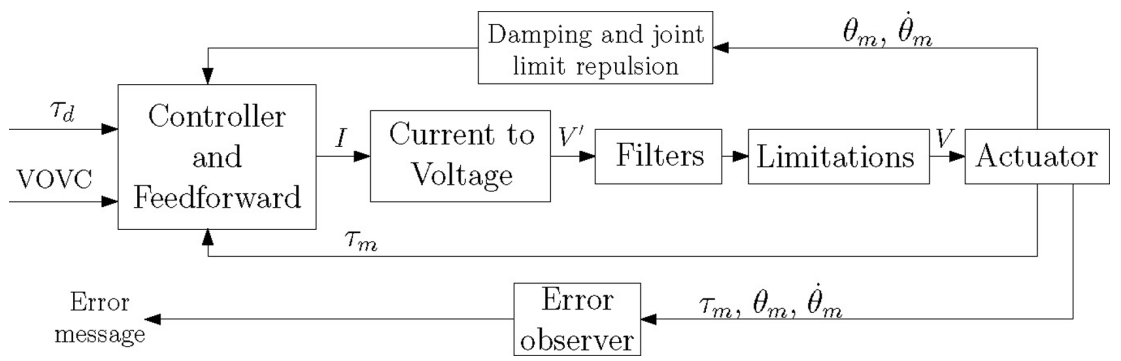


position limit, the actuator will slow down and will be smoothly pushed away from the limitation. If an actuator still reaches its limitation, all actuators will switch back to position control mode and the robot will thus stop.

\section{INTEGRATIONS AND APPLICATIONS}

\section{Integrations}

Kinova's robotic technologies can be integrated to different systems. This section presents official integrations with other robotic products.

\section{Clearpath Robotics Inc.}

Clearpath Robotics Inc. is a global robotics company dedicated to automating the world's dullest, dirtiest, and deadliest jobs with intelligent service robots. The company provides robot hardware, software and services to enable robot development, deployment and operation. Clearpath is serving markets that span industrial materials handling, mining, military, agriculture, aerospace and academia.

Clearpath's robots integrate very well with Kinova products, as shown in Figure 2. With no exterior control boxes and minimal power supply requirements, Clearpath robots easily supply the necessary user power and USB connection required for communications. Clearpath and Kinova products natively support ROS, so the active open source community of developers facilitate integration. Simulation, control and feedback of the arm are constantly evolving.

\section{Adept MobileRobots}

Adept MobileRobots (previously known as ActivMedia Robotics and MobileRobots, Inc.) designs and manufactures intelligent mobile robots as shown in as shown in Figure 15. In 2010, MobileRobots Inc. was acquired by Adept Technology. The Pioneer Manipulator is a rugged, reliable, sophisticated robot purpose built for the research community and its needs. Designed to be extensively capable out of the box in a broad array of applications, the Pioneer Manipulator is sure to immediately enhance new or existing program. The LX base was integrated with a pair of manipulation arms from Kinova that are also aimed at research applications. These manipulators are mounted to a custom designed torso with

Figure 15. Adept MobileRobots pioneer manipulator. Figure courtesy of Adept MobileRobots.

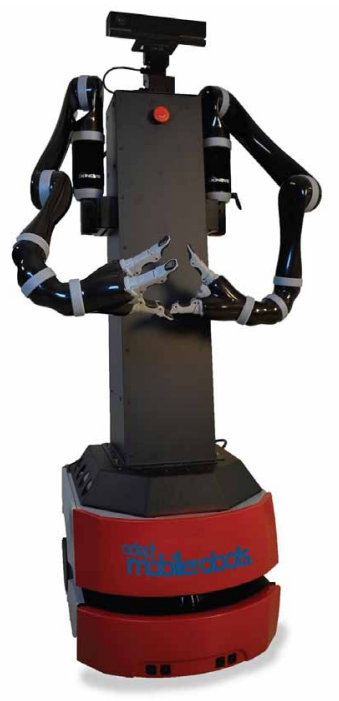


two different mounting points to allow for a higher or lower overall reach depending on your needs. At the top of the torso the Kinect for Windows V2RGB-D Sensor is mounted on a pan/tilt stage allowing for continuous coverage of the workspace while the arms are mounted in either configuration.

\section{Quanser}

Quanser develops experimental plants and courseware for controls, robotics and mechatronics research and teaching. Their open-architecture solutions are used by universities, colleges, research laboratories and commercial organizations. Recently, Quanser expanded its open architecture serial manipulators product line with solutions building on Kinova's MICO robot:

- The Quanser Robotics Package for Education, developed to introduce robotics at the undergraduate level, combines the 4 DOF version of MICO with multi-seat simulation and 3 D visualization and extensive courseware materials;

- The Quanser Robotics Platform for Research gives researchers an open architecture 6 DOF MICO platform, with direct access to each joint in real time. Coupled with simulation and 3D visualization, it allows for quick control algorithm testing, prior to its deployment on the physical system.

Quanser's R\&D engineers also took advantage of the Kinova's robot arms developing the Hapticbased Immersive Telerobotic System for IED Disposal. Using three customized JACO robots they created a dual-arm anthropomorphic and vision arm configuration that allows for intuitive human control of remote arms and camera viewpoints. This project was developed in collaboration with Defence Research and Development Canada, Canadian Safety and Security Program and CBRNE Research and Technology Initiative.

\section{SynTouch}

SynTouch provides a biomimetic tactile sensor with three sensory modalities: deformation, vibration, and temperature, as shown in Figure 16. In collaboration with Kinova, SynTouch has created the BioTac System for JACO. This provides the mechanical and electrical adapters to integrate up to 3 BioTac sensors into each JACO hand. The mechanical adapters replace the distal segment of each modular finger and an internally mounted electrical adapter board aggregates and transmits tactile data through the arm to a computer. Researchers use these sensors to enable projects involving object perception and manipulation, and to improve the utility of robotic systems.

Figure 16. BioTac system for JACO. Figure courtesy of SynTouch.

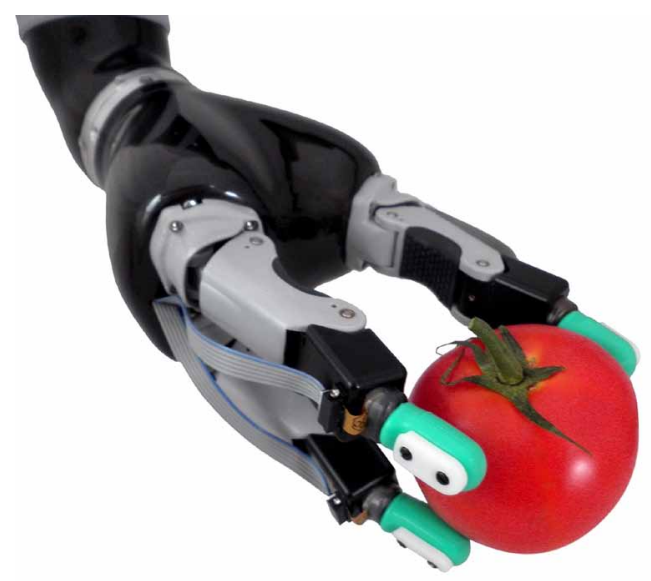




\section{Stanley Robotics}

Stanley Innovation specializes in mobile robotics solutions for commercial and research customers. Their core engineering team branched off from Segway Robotics after leading the advanced development group that created the newest RMP (Robotic Mobility Platform) architecture. They continue to work with the RMP architecture by developing new solutions for customers using RMP as the core mobile platform. For instance, they integrate RMP's with peripheral technologies like Kinova's JACO to deliver a complete solution to end customers, as shown in Figure 17.

\section{Robotnik}

Robotnik is a company specialized in robot product development and R\&D projects. Robotnik has a large experience in mobile platforms and mobile manipulators such as XL-JACO and XL-MICO, shown in Figure 18. Kinova arms provide the mobile platform with direct handling capabilities leading to a robotic system for research and different applications.

\section{Applications / Research}

Kinova's products are used in many applications and research work such as mobile manipulation (Dimitrov et al., 2013; Allan and Beaudry, 2014; Fathzadeh et al., 2014), computer vision algorithms (Jiang et al., 2013), human-robot interaction (Kondaxakis et al., 2014; Leroux et al., 2013), artificial intelligence (Lampe and Riedmiller, 2013), teleoperation (Bidwell et al., 2014), assistance (Cavallo et al., 2014; Maheu et al., 2011), medical applications (Entsfellner et al., 2012; Morse et al., 2013; Entsfellner et al., 2013), planning (Eich et al., 2014), tactile sensing (Lee et al., 2013), control interfaces (Bougrain et al., 2013; Lampe et al., 2014; Bassily et al., 2014; Ianov et al., 2013; Fall et al., 2015) and kinematics (Gosselin and Liu, 2014). Figure 19 shows an integration of JACO at Worcester Polytechnic Institute (WPI) (Dimitrov et al., 2013). The mission of the CARE (Cyber Physical Systems for Advanced Response to Epidemics) project team at WPI is to deliver a safe, reliable, and intuitive emergency treatment unit to facilitate a higher degree of safety and situational awareness for medical staff, leading to an increased level of patient care during an epidemic outbreak in an unprepared,

Figure 17. Stanley robotics ARTI platform. Figure courtesy of Stanley Robotics.

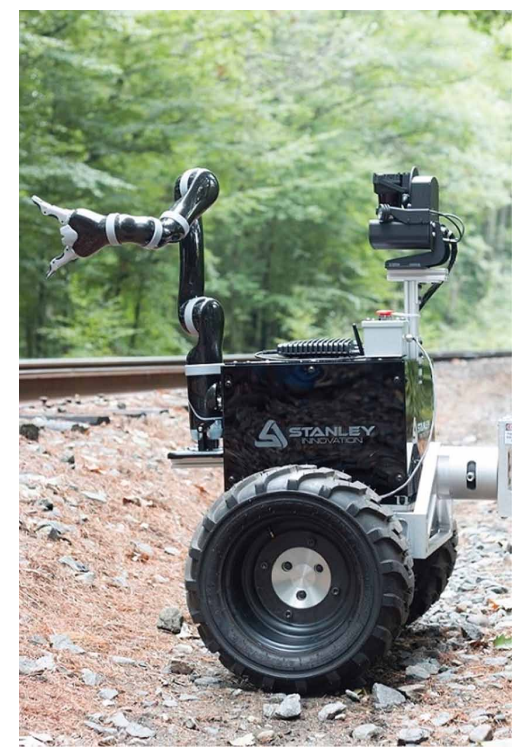


Figure 18. Robotnik XL-MICO. Figure courtesy of Robotnik.

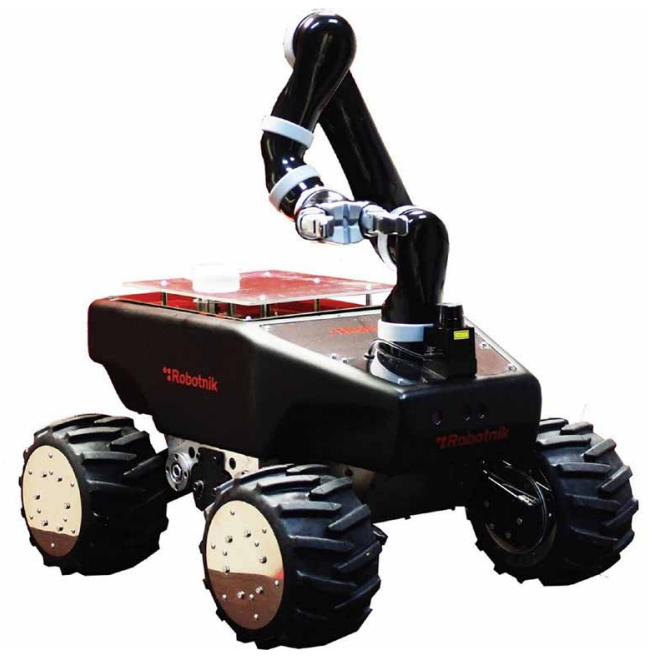

Figure 19. AERO, Autonomous Exploration Rover, with decontamination tanks. Figure courtesy of RIVeR Lab, Worcester Polytechnic Institute.

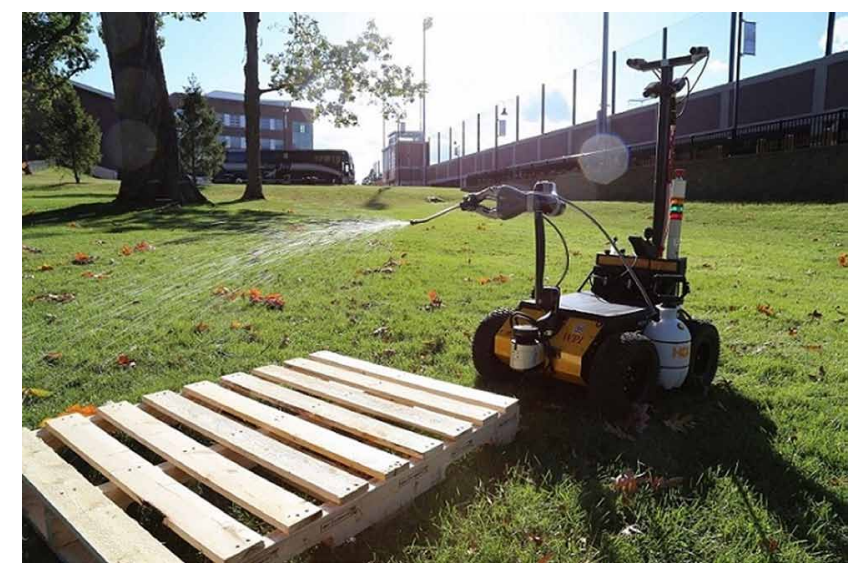

underdeveloped, or disaster stricken area. This includes research on using mobile robots with dexterous manipulators, including the Kinova JACO manipulator, for remote decontamination and inspection activities. Figure 20 shows the Robot-Era project [16]. The domestic robot has several services, including manipulation functionalities. Thanks to the use of the Kinova JACO arm, the robot is able to pick objects from a table and to bring them to the person. Figure 21 shows two JACO in a torso configuration mounted on a grantry for research in human-robot collaboration at the Laboratoire de robotique de l'Université Laval. Figure 22 shows a MICO robot at Carnegie Mellon University being used for research in intelligent algorithms to help enable people living with disabilities to perform activities of daily living and instrumental activities of daily living (Herlant et al., 2016). 
Figure 20. Robot-Era project. Figure courtesy of Scuola Superiore Sant'Anna.

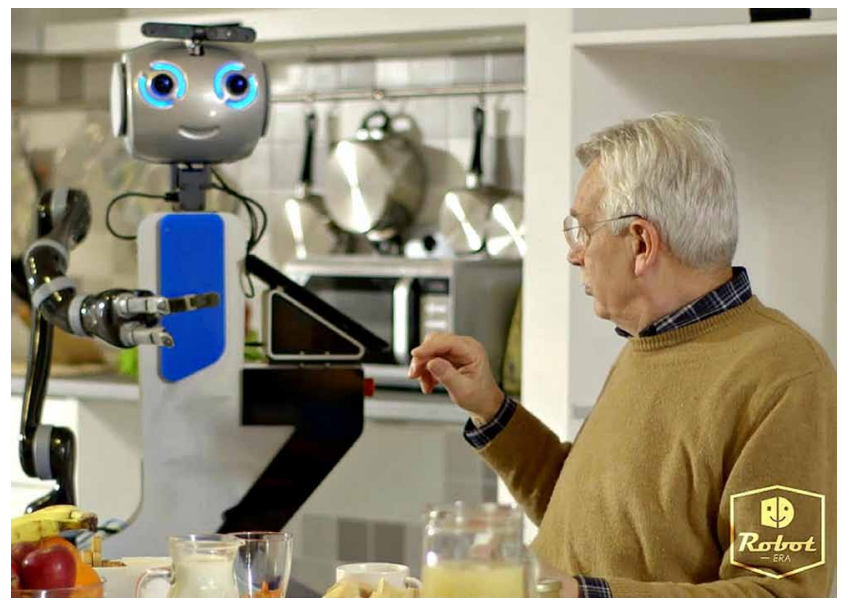

Figure 21. Two JACO in a torso configuration mounted on a grantry. Figure courtesy of Laboratoire de robotique de l'Université Laval.

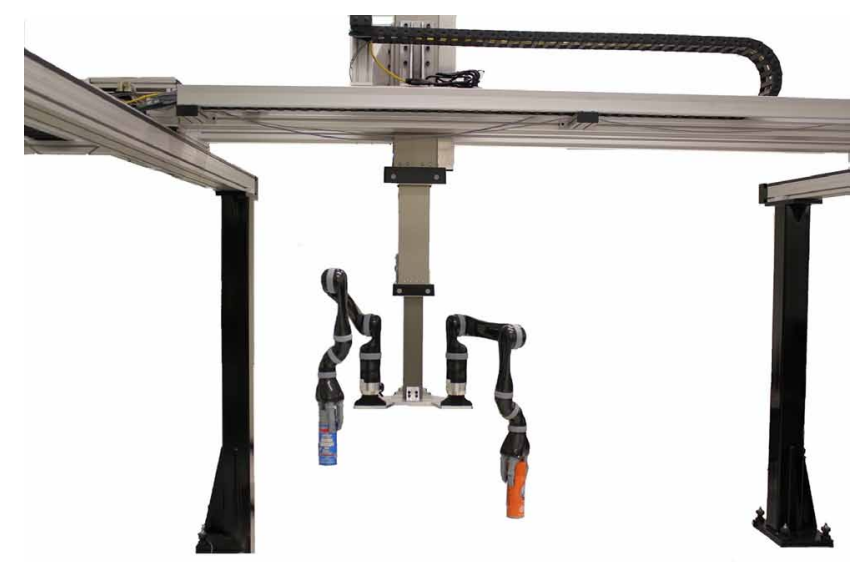

Figure 22. $\mathrm{MICO}^{2}$ robot augmented with a depth camera and computing power being used to autonomously detect, obtain, and deliver a morsel of food to the operator's mouth at Carnegie Mellon University. Figure courtesy of the Personal Robotics Lab at Carnegie Mellon University.

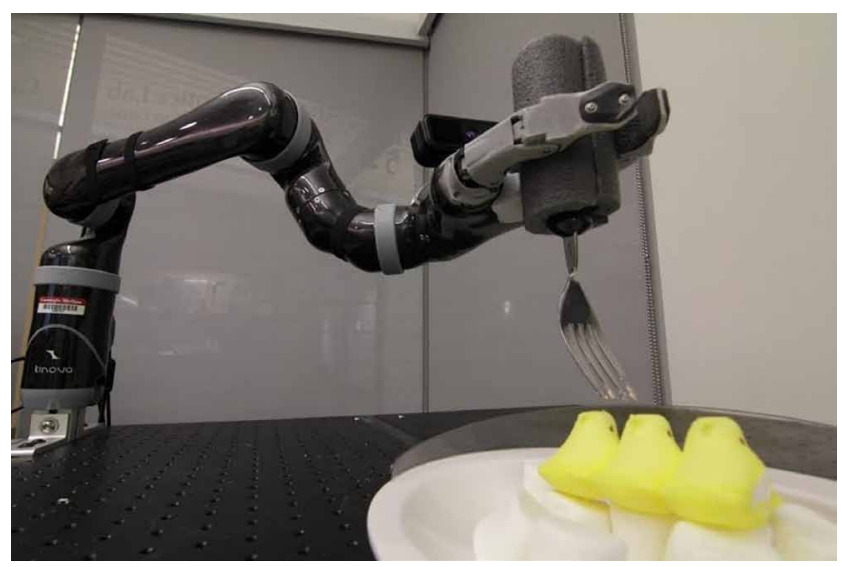




\section{CONCLUSION}

This paper presented Kinova's modular systems. Classic robot configurations such as $\mathrm{JACO}^{2}$ and $\mathrm{MICO}^{2} 6$ DOF and 4 DOF versions were presented along with their specifications. The modular grippers (KG-2 and KG-3) and the modular actuators (K-75+, K-75 and K-58) were then introduced. These hardware systems can also be used to configure custom robotic systems. The different control interfaces to interact with Kinova's products were then detailed. These include a Software Development Kit (SDK) (including a high-level API, a low-level API and a Development center), a ROS package and a Joystick. The control modes, including position, velocity and torque control, were then presented along with their features. Finally, integration of Kinova's technology to other products were presented along with application examples. 


\section{REFERENCES}

Allan, J.-F., \& Beaudry, J. (2014). Robotic systems applied to power substations - A state-of-the-art survey. In Proceedings of the 2014 3rd International Conference on Applied Robotics for the Power Industry (CARPI). doi:10.1109/CARPI.2014.7030049

Bassily, D., Georgoulas, C., Guettler, J., Linner, T., \& Bock, T. (2014). Intuitive and Adaptive Robotic Arm Manipulation using the Leap Motion Controller. In Proceedings of the 41 st International Symposium on Robotics ISR/Robotik'14.

Bidwell, J., Holloway, A., \& Davidoff, S. (2014). Measuring Operator Anticipatory Inputs in Response to Timedelay for Teleoperated Human-robot Interfaces. In Proceedings of the SIGCHI Conference on Human Factors in Computing Systems (pp. 1467-1470). New York, NY, USA: ACM. doi:10.1145/2556288.2557174

Birglen, L., Laliberté, T., \& Gosselin, C. M. (2008). Underactuated Robotic Hands (Vol. 40). Springer.

Bougrain, L., Rochel, O., Boussaton, O., \& Havet, L. (2012). From the decoding of cortical activities to the control of a $\{\mathrm{JACO}\}$ robotic arm: a whole processing chain.

Campeau-Lecours, A., Maheu, V., Lepage, S., Lamontagne, H., Latour, S., Paquet, L., \& Hardie, N. (2016). JACO Assistive Robotic Device: Empowering People With Disabilities Through Innovative Algorithms. In Rehabilitation Engineering and Assistive Technology Society of North America (RESNA) Annual Conference, Washington DC, July.

Cavallo, F., Limosani, R., Manzi, A., Bonaccorsi, M., Esposito, R., Di Rocco, M., \& Dario, P. et al. (2014). Development of a Socially Believable Multi-Robot Solution from Town to Home. Cognitive Computation, 6(4), 954-967. doi:10.1007/s12559-014-9290-z

De Santis, A., Siciliano, B., De Luca, A., \& Bicchi, A. (2008). An atlas of physical human--robot interaction. Mechanism and Machine Theory, 43(3), 253-270. doi:10.1016/j.mechmachtheory.2007.03.003

Dimitrov, V., DeDonato, M., Panzica, A., Zutshi, S., Wills, M., \& Padir, T. (2013). Hierarchical Navigation Architecture and Robotic Arm Controller for a Sample Return Rover. In Proceedings of the 2013 IEEE International Conference on Systems, Man, and Cybernetics (SMC) (pp. 4476-4481). doi:10.1109/SMC.2013.761

Eich, M., Hartanto, R., Kasperski, S., Natarajan, S., \& Wollenberg, J. (2014). Towards Coordinated Multirobot Missions for Lunar Sample Collection in an Unknown Environment. Journal of Field Robotics, 31(1), 35-74. doi:10.1002/rob.21491

Entsfellner, K., Strauss, G., Berger, T., Dietz, A., \& Lueth, T. C. (2012). Micro-Macro Telemanipulator for Middle-Ear Microsurgery. In Proceedings of the 7th German Conference on Robotics ROBOTIK '12 (pp. 1-4).

Entsfellner, K., Tauber, R., Roppenecker, D. B., Gumprecht, J. D. J., Strauss, G., \& Lueth, T. C. (2013). Development of universal gripping adapters: Sterile coupling of medical devices and robots using robotic fingers. In Proceedings of the 2013 IEEE/ASME International Conference on Advanced Intelligent Mechatronics (AIM) (pp. 1464-1469). doi:10.1109/AIM.2013.6584301

Fall, C. L., Turgeon, P., Campeau-Lecours, A., Maheu, V., Boukadoum, M., Roy, S., . . Gosselin, B. (2015). Intuitive Wireless Control of a Robotic Arm for people living with an upper body Disability. In Proceedings of the 37th Annual International Conference of the IEEE Engineering in Medicine and Biology Society (EMBC'15), Milano, Italy. doi:10.1109/EMBC.2015.7319370

Fathzadeh, R., Mokhtari, V., Abdollahi, F., Nabavi, N., Abazari, F., \& Bagheri, H. (2014). Mobile robot in domestic environment. In Proceedings of the 2014 International Conference on Information Science, Electronics and Electrical Engineering (ISEEE) (Vol. 2, pp. 918-922). doi:10.1109/InfoSEEE.2014.6947802

Gosselin, C., \& Liu, H. (2014). Polynomial inverse kinematic solution of the Jaco robot. In ASME International Design Engineering Technical Conferences and Computers and Information in Engineering Conference. doi:10.1115/DETC2014-34152

Haddadin, S., \& Croft, E. (2016). Physical human-robot interaction. In Handbook of Robotics (pp. 1835-1874). Springer.

Hannaford, B., \& Ryu, J.-H. (2002). Time-domain passivity control of haptic interfaces. IEEE Transactions on Robotics and Automation, 18(1), 1-10. doi:10.1109/70.988969 
Herlant, L. V., Holladay, R. M., \& Srinivasa, S. S. (2016). Assistive Teleoperation of Robot Arms via Automatic Time-Optimal Mode Switching. In Proceedings of the ACM/IEEE International Conference on Human Robot Interaction (pp. 35-42). doi:10.1109/HRI.2016.7451731

Ianov, A. I., Kawamoto, H., \& Sankai, Y. (2013). Development of noise resistant hybrid capacitive-resistive electrodes for wearable robotics, computing and welfare. In Proceedings of the IEEE/RSJ International Conference on Intelligent Robots and Systems (IROS) (pp. 4249-4254). doi:10.1109/IROS.2013.6696965

Jiang, H., Wachs, J. P., \& Duerstock, B. S. (2013). Integrated vision-based robotic arm interface for operators with upper limb mobility impairments. In Proceedings of the 2013 IEEE International Conference on Rehabilitation Robotics. doi:10.1109/ICORR.2013.6650447

Kondaxakis, P., Pajarinen, J., \& Kyrki, V. (2014). Real-time recognition of pointing gestures for robot to robot interaction. In Proceedings of the 2014 IEEE/RSJ International Conference on Intelligent Robots and Systems (IROS 2014) (pp. 2621-2626). doi:10.1109/IROS.2014.6942920

Lampe, T., Fiederer, L. D. J., Voelker, M., Knorr, A., Riedmiller, M., \& Ball, T. (2014). A Brain-computer Interface for High-level Remote Control of an Autonomous, Reinforcement-learning-based Robotic System for Reaching and Grasping. In Proceedings of the 19th International Conference on Intelligent User Interfaces (pp. 83-88). New York, NY, USA: ACM. doi:10.1145/2557500.2557533

Lampe, T., \& Riedmiller, M. (2013). Acquiring visual servoing reaching and grasping skills using neural reinforcement learning. In Proceedings of the 2013 International Joint Conference on Neural Networks (IJCNN). doi:10.1109/IJCNN.2013.6707053

Lecours, A., \& Gosselin, C. (2013). Computed-Torque Control of a Four-Degree-of-Freedom Admittance Controlled Intelligent Assist Device. In Experimental Robotics (Vol. 88, pp. 635-649). Springer International Publishing. doi:10.1007/978-3-319-00065-7_43

Lecours, A., Mayer-St-Onge, B., \& Gosselin, C. (2012). Variable admittance control of a four-degree-of-freedom intelligent assist device. In Proceedings of the 2012 IEEE International Conference on Robotics and Automation (pp. 3903-3908). IEEE. doi:10.1109/ICRA.2012.6224586

Lee, W. W., Cabibihan, J., \& Thakor, N. V. (2013). Bio-mimetic strategies for tactile sensing. IEEE Sensors. doi:10.1109/ICSENS.2013.6688260

Leroux, C., Lebec, O., Ben Ghezala, M. W., Mezouar, Y., Devillers, L., \& Chastagnol, C. ... Fattal, C. (2013). ARMEN: Assistive robotics to maintain elderly people in natural environment. IRBM, 34(2), 101-107.

Maheu, V., Frappier, J., Archambault, P. S., \& Routhier, F. (2011). Evaluation of the JACO robotic arm: Clinicoeconomic study for powered wheelchair users with upper-extremity disabilities. In Proceedings of the 2011 IEEE International Conference on Rehabilitation Robotics (ICORR), (pp. 1-5). doi: $<$ ALIGNMENT.qj $></$ ALIGNMENT>10.1109/ICORR.2011.5975397

Morse, J., Wehbe, M., Taddei, R., Cyr, S., \& Hemmerling, T. M. (2013). Magellan: Technical description of a new system for robot-assisted nerve blocks. Journal of Computers, 8(6), 1401-1405. doi:10.4304/jcp.8.6.1401-1405

Villani, L., \& De Schutter, J. (2008). Force Control. In B. Siciliano \& O. Khatib (Eds.), Springer Handbook of Robotics (pp. 161-185). Springer Berlin Heidelberg. doi:10.1007/978-3-540-30301-5_8 
Alexandre Campeau-Lecours is a professor at Université Laval. His main expertise is in control algorithms and robotics. His work focuses mainly on physical human-robot interaction and medical devices. Prior to joining Université Laval, he was project manager in robotics and control at Kinova.

Hugo Lamontagne was born in Canada in 1981. He received his bachelor's degree in 2009 in software engineering from Ecole de Technologie Superieure. In 2010, he joined Kinova where he developed control interfaces for various products. He is currently responsible for the software interfaces/API development of the different robotic arms at Kinova.

Simon Latour, was born in Canada in 1981. He received his bachelor's degree in 2009 in electrical engineering with specialization in medical technologies at Ecole de Technologie Superieure. From 2005 to 2010 he worked for Telemedic where he did research on the integration of pulse oxymetry sensors for portable devices and worked on the implementation of communication interfaces for medical devices. In 2010, he joined Kinova where he developed a control interface that allows robot arms to be used with multiple powered wheelchair's controller (including joysticks, head array, sip and puff controls). He is currently responsible for the firmware development of the different robotic arms at Kinova.

Philippe Fauteux received his Bachelor's degree in mechanical engineering in 2007 and his Master's degree in mechanical engineering in 2010 from the Université de Sherbrooke, Québec, Canada. He was at research department of Maxon Motor in 2010 and 2011 to work on the development of novel actuators architectures. In 2012, he joined the product development team at Kinova Robotics.

Veronique Maheu was born in Laval in 1982. After technical studies in aeronautics, she completed a bachelor degree in mechanical engineering and a master degree in engineering, specialized in health technology in 2011. The master project was about treating and assessing the use of electromyographic signals in spinal cord injury subject to control a robotic manipulator. In the following years, she has worked for Kinova Robotics where she managed research and technical partnership in addition to the local distribution division. In 2013, she joined forces to create the company's knowledge center and training strategy for users and distributors.

François Boucher is the Chief Business Development Officer at Kinova Inc. in Montreal, Canada. He received his MBA and a Bachelor degree in Physics Engineering from Laval University. After his graduation, he worked as the General Manager of a technology transfer and investment company before joining Kinova in its early days to develop the service robotics market. In 2014 and 2015 Kinova was listed as one of the 50 most influential public \& private companies in the global robotics industry by the Robotics Business Review. Through his passion for robotics, he continues to have an eye towards identifying new opportunity and driving business growth and requirements for product development.

Charles Deguire received his bachelor's degree in 2007 in electrical engineering with specialization in medical technologies at École de Technologie Supérieure, Montreal, Canada. In 2006, he co-founded Kinova where he is now the Chief Executive Officer.

Louis-Joseph Caron L'ecuyer received his bachelor's degree in 2007 in electrical engineering with specialization in medical technologies at École de Technologie Supérieure, Montreal, Canada. In 2006, he co-founded Kinova where he is now the Chief Technical Officer. 